(D) Dixis Figueroa Pedraza ${ }^{1}$

(D)Anahi Cézar de Lima Lins ${ }^{1}$

(D) Eduarda Emanuela Silva dos Santos ${ }^{1}$

(D) Maria Mônica de Oliveira ${ }^{1}$

1 Universidade Estadual da Paraíba. Campina Grande, PB, Brasil.

Correspondência Dixis Figueroa Pedraza dixisfigueroa@gmail.com

\section{Década de Ação em Nutrição: reflexões sobre a conjuntura brasileira}

\author{
Decade of Action in Nutrition: reflections on the Brazilian \\ conjuncture
}

\begin{abstract}
Resumo
Objetivo: Fazer uma reflexão sobre a conjuntura brasileira relacionada à Década de Ação em Nutrição. Métodos: Ensaio desenvolvido por meio de pesquisa teórica de base bibliográfica. Resultados: A Agenda 2030 surgiu como alternativa para discutir maneiras de melhorar o quadro de necessidades humanas e as condições do meio ambiente de maneira sustentável. A nutrição tem papel de destaque nesse sentido, sendo ao menos 12 dos Objetivos de Desenvolvimento Sustentável (ODS) relacionados ao estado nutricional. Nesse contexto, a Década de Ação em Nutrição está permeada em seis dos 17 ODS e focada no segundo que visa acabar com a fome, alcançar a segurança alimentar e nutricional, melhorar a nutrição e promover a agricultura sustentável. Como signatário da Década de Ação em Nutrição, o Brasil declarou, por meio de seis pilares, seu acordo internacional com a melhoria da qualidade da alimentação da sua população e com a promoção de um sistema alimentar sustentável. Este ensaio destaca os pilares segundo e terceiro, que enfocam a importância da cobertura universal de ações em nutrição nos sistemas de saúde e da proteção social. Realiza-se uma reflexão acerca da incorporação das ações de nutrição no sistema de saúde brasileiro e da relevância do Programa Bolsa Família na proteção social de grupos vulneráveis no país. Conclusão: Verifica-se como desafios a atuação do nutricionista, o trabalho interdisciplinar e a capacitação dos profissionais de saúde na implantação das ações de alimentação e nutrição nos serviços de saúde. Destaca-se a necessidade de mudanças estruturais no Programa Bolsa Família.
\end{abstract}

Palavras-chave: Nutrição em Saúde Pública. Programas e Políticas de Nutrição e Alimentação. Desenvolvimento Sustentável. Política Pública

\begin{abstract}
Objective: To reflect on the situation of Brazil in relation to the Decade of Action on Nutrition. Methods: Essay developed through bibliographical theoretical research. Results: The Agenda 2030 emerged as an alternative to discuss ways to improve the human needs and environmental conditions in a sustainable way. Nutrition has a prominent role in this regard, with at least 12 of the Sustainable Development Goals (SDGs) being related to nutritional status. In this context, the Decade of Action on Nutrition is permeated in six of the 17 SDGs and is the focus of the second that aims to end hunger, achieve food and nutrition security, improve nutrition and promote sustainable agriculture. As a signatory of the Decade of Action on Nutrition, Brazil declared through six pillars its international agreement with the improvement of food quality of its population and with the promotion of a sustainable food system. The present essay highlights the second and third pillars, which focus on the importance of universal coverage of nutrition actions in health and social protection systems. A
\end{abstract}


reflection is made about the incorporation of nutrition actions in the Brazilian health system and the relevance of the Family Allowance (Bolsa Familia) Program for the social protection of vulnerable groups in the country. Conclusion: The challenges identified were the work of nutritionists, interdisciplinary work, and training of health professionals in the implementation of food and nutrition actions in health services. The need for structural changes in the Bolsa Familia Program is highlighted.

Keywords: Nutrition in Public Health. Nutrition and Food Programs and Policies. Sustainable development. Public policy. 


\section{INTRODUÇÃO}

Combater a má nutrição em todas as suas formas é um dos maiores desafios de saúde em todo o mundo. ${ }^{1}$ A má nutrição materna e infantil é problema global, com consequências relevantes para a sobrevivência, a incidência de doenças agudas e crônicas, o desenvolvimento saudável e a produtividade econômica de indivíduos e sociedades. ${ }^{2}$ Ao alcançar a adequada nutrição, espera-se que ocorram benefícios durante todo o curso da vida, tais como: i) redução da morbimortalidade na infância e incremento no desenvolvimento motor, cognitivo e socioafetivo; ii) melhoria no desempenho social e na capacidade de aprendizado; iii) aumento da estatura do adulto e diminuição da obesidade e doenças crônico-degenerativas; e iv) acréscimo na capacidade de trabalho e produtividade. ${ }^{2,3}$

Assim este artigo, na forma de ensaio, visa fazer uma reflexão sobre a conjuntura brasileira relacionada à Década de Ação em Nutrição (2016-2025). Trata-se de uma pesquisa teórica de base bibliográfica. Para fins didáticos, o texto foi dividido em quatro seções que se complementam em uma tentativa de abordagem integral do tema: a Agenda 2030 para o Desenvolvimento Sustentável; a Década de Ação em Nutrição; a situação nutricional da população brasileira relacionada às metas estabelecidas para a Década de Ação em Nutrição e o compromisso brasileiro para a Década de Ação em Nutrição, com foco na cobertura universal de ações em nutrição nos sistemas de saúde e na proteção social e educação alimentar e nutricional.

\section{Agenda 2030 para o Desenvolvimento Sustentável}

As disparidades de saúde estão presentes desde o início da vida e são influenciadas por diversos fatores, especialmente os socioeconômicos. À medida que essas desigualdades se acumulam no decorrer da vida, restringem o pleno envolvimento do indivíduo em todos os extratos sociais. ${ }^{4}$ A saúde, nesse sentido, é condição prévia, resultado e utilizada como indicador das três dimensões (ambiental, social e econômica) do desenvolvimento sustentável. ${ }^{5}$

Nesse contexto, a Agenda 2030 surgiu como resultado histórico de encontros entre países para discutir maneiras de melhorar o quadro de necessidades humanas e as condições do meio ambiente de maneira sustentável. ${ }^{6}$ Em 1992, durante a Conferência das Nações Unidas sobre Meio Ambiente e Desenvolvimento, representantes de vários países se reuniram no Brasil, na cidade do Rio de Janeiro, para elaborar um plano de ação com o objetivo de garantir às gerações futuras um desenvolvimento sustentável. ${ }^{7}$ Posteriormente, no ano 2000, a Organização das Nações Unidas (ONU) assumiu o compromisso de desenvolver ações de caráter global para a redução da extrema pobreza e da fome, bem como outras privações generalizadas, tornando o tema uma prioridade na agenda internacional de desenvolvimento. Declararam-se, assim, oito Objetivos de Desenvolvimento do Milênio (ODM) que deveriam ser revistos no prazo de 15 anos. ${ }^{8}$

Em 2012, a Conferência das Nações Unidas sobre Desenvolvimento Sustentável - Rio+20 estabeleceu uma nova agenda de desenvolvimento sustentável, caracterizando-se como um importante ponto de partida para a construção do futuro que queremos. No evento foram firmados novos acordos visando ao crescimento econômico e consciente, à proteção ambiental compartilhada, à inclusão social e à erradicação da pobreza. ${ }^{9}$ Posteriormente, na Assembleia Geral da ONU de 2015, celebrada em Nova York, o desenvolvimento sustentável foi definido como "a satisfação das necessidades da atual geração sem comprometer a capacidade de satisfação das necessidades das gerações futuras" como parte do documento "Transformando Nosso Mundo: a Agenda 2030 para o Desenvolvimento Sustentável". 5 
A Agenda 2030 contém 17 Objetivos de Desenvolvimento Sustentável (ODS) e 169 metas. Este conjunto de objetivos reflete uma agenda inovadora, universal e transformadora, baseada nos ODM, para promover o desenvolvimento sustentável integrando suas dimensões econômica, social e ambiental. A Agenda propõe ações voltadas para a segurança do planeta e da população, numa aliança na qual todos os países envolvidos são responsáveis por atingir todos os objetivos até o ano 2030, envolvendo temáticas diversificadas como erradicação da pobreza, segurança alimentar e nutricional e agricultura, saúde, educação, igualdade de gênero, redução das desigualdades, energia, água e saneamento, padrões sustentáveis de produção e de consumo, mudança do clima, cidades sustentáveis, proteção e uso sustentável dos oceanos e dos ecossistemas terrestres, crescimento econômico inclusivo, infraestrutura e industrialização, governança, e meios de implementação. 6,8

O terceiro ODS tem como foco principal a saúde da população, incluindo o acesso aos sistemas de saúde e as condições relacionadas à saúde materno-infantil. Destaca a diminuição da mortalidade relacionada ao nascimento, inclusive às mortes evitáveis, como prioridades de saúde global. ${ }^{10}$ No Brasil, o número de óbitos maternos por mil nascidos vivos diminuiu de 73,3 em 2000 para 64,8 em 2011 (http://tabnet.datasus.gov.br/cgi/idb2012/C03b.htm, acessado em 05/jun/2019), sendo a meta estabelecida pelos ODS de 70 mortes. ${ }^{10}$ Em relação à meta de mortalidade neonatal fixada em 12 mortes a cada mil nascidos vivos, ${ }^{10}$ o Brasil destaca-se por ter atingido o índice de 10,6 em 2011, que era de 16,7 no ano 2000 (http://tabnet.datasus.gov.br/cgi/idb2012/c0104b.htm, acessado em 05/jun/2019). Para o mesmo período, no país verificou-se redução da taxa de mortalidade infantil de 30,1 para 17,7 (http://tabnet.datasus.gov.br/cgi/idb2012/c16b.htm, acessado em 05/jun/2019), quando a meta global estabelecida para 2030 é de 25 mortes por mil nascidos vivos. ${ }^{10}$ Esses avanços colocam o Brasil em posição privilegiada em relação aos indicadores materno-infantis supracitados nos ODS, destacando-se por possuir um sistema de saúde público, universal e gratuito. ${ }^{11}$ Enquanto projeto ético-político de caráter público voltado à proteção social, o Sistema Único de Saúde (SUS) tem representado uma profunda mudança na saúde da sociedade brasileira, assumindo a saúde como produção social, com responsabilidade do Estado e valorização da participação social. ${ }^{12}$

Nesse sentido, a Atenção Primária à Saúde (APS) destaca-se por propiciar progressos na cobertura universal de saúde, nos resultados de saúde e nos ODS, com papel vital nas reduções na mortalidade pósneonatal e infantil..$^{13}$ Como ação prioritária na consolidação, qualificação e expansão da APS, ${ }^{14}$ a Estratégia Saúde da Família (ESF) tem colaborado com melhorias na saúde da criança e na redução na taxa de mortalidade infantil. Ainda, tem representado importante progresso na atenção ao pré-natal e na prevenção de complicações na gestação. ${ }^{15,16}$ Mais recentemente, avaliações específicas da ESF no contexto do Programa Mais Médicos têm destacado contribuições do mesmo na diminuição das internações por condições sensíveis à atenção primária, além da ampliação da assistência na atenção básica, efetivação do direito à saúde e avanços do ponto de vista médico (formação, processo de trabalho e rotatividade). ${ }^{17,18}$

Entretanto, cabe destacar que ainda é de suma importância lidar com questões que têm se mostrado de difícil solução, como o aumento contínuo de doenças crônicas não transmissíveis (DCNT) e a baixa cobertura substancial de saúde das classes mais vulneráveis. Além disso, devem ser citados os problemas de coordenação da APS que incluem a falta de comunicação com os demais serviços, as barreiras de acesso aos serviços especializados e o sentimento de descontinuidade assistencial pelos usuários. ${ }^{11,12}$ 


\section{Década de Ação em Nutrição}

A nutrição tem papel de destaque no desenvolvimento sustentável, sendo estabelecida com indicadores do estado nutricional ao menos em 12 dos ODS. Condições adequadas de nutrição podem potencializar os ODS, por se configurar em benefícios para a saúde, educação, emprego, empoderamento feminino e redução da pobreza e da desigualdade. ${ }^{19}$ Consideram-se cinco âmbitos fundamentais dos ODS, para os quais a nutrição pode contribuir e dos quais pode beneficiar-se: i) produção sustentável de alimentos; ii) sistemas de infraestrutura sólidos; iii) sistemas de saúde; iv) equidade e inclusão; e v) paz e estabilidade das nações. Dessa forma, melhorar a nutrição e erradicar a má nutrição em todas as suas formas é essencial à consolidação dos ODS. ${ }^{20}$

Reafirmando os compromissos da Agenda 2030 e a nutrição como eixo central do desenvolvimento sustentável, a ONU proclamou recentemente a Década de Ação em Nutrição (2016-2025). O objetivo é coordenar esforços globais, com efetiva participação das nações, para o enfrentamento de todas as formas de má nutrição (sobrepeso e obesidade, problemas de crescimento, desnutrição crônica e deficiência de micronutrientes). ${ }^{21}$ A Década de Ação em Nutrição está permeada em seis dos 17 ODS e focada no segundo objetivo, que visa acabar com a fome, alcançar a segurança alimentar e nutricional, melhorar a nutrição e promover a agricultura sustentável. ${ }^{22}$

Assim, foram estabelecidas como metas globais da nutrição para 2025: i) reduzir em 40\% o número de crianças menores de cinco anos com déficit de estatura; ii) reduzir em 50\% a anemia em mulheres em idade reprodutiva; iii) reduzir em 30\% o baixo-peso ao nascer; iv) garantir que não haja aumento do excesso de peso em crianças; v) aumentar em pelo menos 50\% a taxa de amamentação exclusiva nos primeiros seis meses de vida; e vi) reduzir para menos de $5 \%$ o número de crianças com baixo-peso para altura. ${ }^{21}$

\section{Situação nutricional da população brasileira relacionada às metas estabelecidas para a Década de Ação em Nutrição}

O Brasil, segundo dados nacionais, destaca-se por ter apresentado alguns progressos no enfrentamento de todas as formas de má nutrição e, portanto, no alcance das metas globais da nutrição para 2025. O país conseguiu reduzir a fome para menos de $5 \%$ e diminuiu pela metade o número e a porcentagem de desnutridos. No entanto, a anemia entre as mulheres em idade reprodutiva e em crianças menores de cinco anos apresenta prevalências $\geq 5 \%$, constituindo importante problema de saúde pública. Da mesma forma, em relação ao aumento da obesidade, considera-se que o Brasil possivelmente não alcançará a meta até 2025. ${ }^{20,23}$ Além disso, observam-se desigualdades regionais e grupos vulneráveis desfavorecidos, nos quais perpetuam injustiças sociais e manifestam-se paradoxos entre os excessos e carências nutricionais. ${ }^{23}$

No que concerne à situação de segurança alimentar e nutricional, a Pesquisa Nacional por Amostra de Domicílios (PNAD) de 2013 revelou que, na época do estudo, 14,8\% dos domicílios brasileiros estavam em insegurança alimentar e nutricional leve; 4,6\% em situação moderada e 3,2\% grave. As regiões Norte (36,1\%) e Nordeste $(38,1 \%)$ apresentaram as maiores prevalências de domicílios em insegurança alimentar e nutricional, mostrando grandes disparidades com as regiões Sudeste (14,5\%), Sul (14,9\%) e Centro-Oeste (18,2\%). A zona rural da Região Nordeste foi a que teve o percentual mais elevado de domicílios em situação de insegurança alimentar e nutricional moderada ou grave - de 20,1\%. Ainda, os resultados evidenciaram que $78,9 \%$ dos domicílios em insegurança alimentar e nutricional moderada ou grave pertenciam à classe de até um salário-mínimo. ${ }^{24}$ Achados similares foram encontrados por outros pesquisadores que constataram que quanto menor a renda mensal domiciliar por pessoa, maior o número de domicílios em 
situação de insegurança alimentar e nutricional moderada ou grave. ${ }^{25}$ Resultados de uma revisão sistemática da literatura revelaram a determinação social da insegurança alimentar e nutricional no Brasil, destacando as famílias atendidas em serviços públicos de saúde/beneficiárias do Programa Bolsa Família (PBF) com maiores chances de insegurança alimentar e nutricional, quando considerados como referências estudos de base populacional e os resultados da PNAD. ${ }^{26}$

Em relação ao estado nutricional, cujos dados nacionais mais recentes são de 2006, destaca-se que no Brasil a desnutrição aguda nas crianças menores de cinco anos não constitui problema de saúde pública, apresentando declínio modesto de 2,2\% em 1996 para 1,5\% em 2006. A Região Nordeste foi a que apresentou melhor evolução, de 3,4\% para 1,7\% nesse período. ${ }^{27}$ Contudo, os dados do SISVAN Web apontam prevalências do desfecho que oscilaram entre 4,9\% e 6,0\% no período de 2008 a 2019 (Relatórios Públicos. http://sisaps.saude.gov.br/sisvan/relatoriopublico/index, acessado em 26/jan/2020).

Por sua vez, o déficit de estatura persiste como agravo nutricional, apesar da redução acentuada entre 1996 (13,5\%) e 2006 (7,1\%), sobretudo na Região Nordeste, que apresentou o maior declínio, de 22,1\% em 1996 para 5,9\% em 2006. ${ }^{28}$ Entretanto, mostram-se resultados desiguais, com altas prevalências de baixa estatura em crianças de grupos vulneráveis da população como indígenas (26\%), quilombolas (16\%), beneficiários do PBF (15\%) e residentes na Região Norte (15\%). ${ }^{29}$ Segundo a Pesquisa de Orçamentos Familiares de 2008-2009, a redução do déficit de estatura relaciona-se com o aumento da renda (8,2\% no estrato de menor renda para 3,1\% no estrato de maior renda). ${ }^{30}$ Segundo os dados do SISVAN Web, entre 2008 e 2019, déficits de crescimento linear estavam presentes entre 12,3\% e 15,1\% dos menores de cinco anos (Relatórios Públicos. http://sisaps.saude.gov.br/sisvan/relatoriopublico/index, acessado em 26/jan/2020).

Por fim, o excesso de peso nas crianças brasileiras menores de cinco anos de idade mostrou estabilidade entre 1996 e 2006 (cerca de 7,0\% em ambos os anos). Para as diferentes regiões do país, no mesmo período, o maior aumento na prevalência ocorreu no Sul, de 6,9\% e 9,0\%, enquanto no Nordeste foi de 6,9\% e 7,1\%.27 Do ponto de vista de desigualdades sociais, pesquisadores mostraram tendência linear inversa entre o nível socioeconômico e o excesso de peso no caso das crianças do sexo feminino. ${ }^{31}$ Utilizando dados secundários do SISVAN Web, o sobrepeso/obesidade oscilou entre 14,1\% e 17,3\% nos anos de 2008 a 2019 (Relatórios Públicos. http://sisaps.saude.gov.br/sisvan/relatoriopublico/index, acessado em 26/jan/2020).

Quanto aos dados de prevalência apresentados, é possível destacar que os registrados por meio do SISVAN Web sugerem proporções maiores de má nutrição às encontradas em 2006, no período de 2008 a 2019, sinalizando a melhoria do estado nutricional das crianças como um desafio para as políticas públicas que ainda perpetua. Dessa forma, analisar a conjuntura brasileira relacionada à garantia da segurança alimentar e nutricional, conforme preconizado na Década de Ação em Nutrição, reverte-se de vital importância, sobretudo no atual contexto político do país, caracterizado por um período de enfraquecimento ou desmonte das políticas de proteção social, de saúde e de fortalecimento dos sistemas alimentares sustentáveis e promotores de alimentação saudável; bem como pelo questionamento dos mecanismos de enfrentamento da obesidade, com deterioração das condições de vida e do aumento da pobreza, a partir de 2016, precedido por um período em que as políticas em foco foram priorizadas na agenda governamental. ${ }^{32,33}$ Nesse sentido, as políticas relacionadas à expansão dos cuidados básicos de saúde e ao aumento do poder aquisitivo/renda familiar nos lares de baixo poder aquisitivo ganham destaque ao ponderar que esses fatores, junto à melhoria da escolaridade materna e à expansão da rede pública de saneamento básico, foram as principais razões que explicaram o declínio da desnutrição no país entre 1996 e 2007.34 


\section{Compromisso brasileiro para a Década de Ação em Nutrição}

Como signatário da Década de Ação em Nutrição, o Brasil declarou seu acordo internacional com a melhoria da qualidade da alimentação da sua população e com a promoção de um sistema alimentar sustentável, por meio de compromissos convergentes com as metas previamente assumidas no Plano Nacional de Segurança Alimentar e Nutricional, com prazo de cumprimento até 2019. Dessa forma, o Brasil apresenta sua convenção em seis pilares: i) sistemas alimentares sustentáveis e promotores de alimentação saudável; ii) cobertura universal de ações em nutrição nos sistemas de saúde; iii) proteção social e educação alimentar e nutricional; iv) comércio e investimento para melhor nutrição; v) ambientes seguros e propícios para a nutrição em todas as idades; e vi) revisão, fortalecimento e promoção da governança em nutrição e prestação de contas. $^{22}$

O primeiro pilar postulado no Brasil para a Década de Ação em Nutrição ressalta a relação de causalidade entre sistemas alimentares sustentáveis e a promoção da alimentação saudável, reforçando a importância da integração entre as políticas de nutrição, alimentação e agricultura. Nesse sentido, a promoção da produção de alimentos saudáveis e sustentáveis e a estruturação da agricultura familiar com incentivos governamentais devem ser consideradas, dada a importância como metas para a alimentação saudável e os sistemas alimentares sustentáveis. Com destaque, propõe-se incrementar anualmente os recursos financeiros transferidos pelo Programa Nacional de Alimentação Escolar e destinados à aquisição de alimentos provenientes da agricultura familiar. ${ }^{22}$

O segundo e terceiro pilares representam o foco principal do presente ensaio. Tratam, respectivamente, sobre a inclusão das intervenções nutricionais nos sistemas de saúde e nos mecanismos de proteção social. ${ }^{22}$

O quarto pilar foca o papel do comércio para o alcance das metas globais. O pilar nº 5 dá ênfase à importância do ambiente (doméstico, escolar, laboral e institucional) na ocorrência de todas as formas de má nutrição. O pilar seis inclui a necessidade do monitoramento permanente no que tange ao alcance das metas globais de alimentação e nutrição, a importância de políticas e programas que impactem a nutrição e a necessidade de cooperação entre países nas questões nutricionais. ${ }^{22}$

\section{Cobertura universal de ações em nutrição nos sistemas de saúde}

O segundo pilar postulado no Brasil para a Década de Ação em Nutrição destaca a importância de incorporar as ações de nutrição nos sistemas de saúde.22 Por entender que a alimentação é um fator condicionante e determinante da saúde, as ações de alimentação e nutrição devem ser organizadas para atender às demandas geradas pelos agravos relacionados à má alimentação dentro do cuidado integral na rede de atenção à saúde. ${ }^{29} \mathrm{O}$ desenvolvimento de tais ações, de maneira qualificada e multidisciplinar, é essencial para a garantia dos princípios do SUS (integralidade, universalidade e resolubilidade) no contexto da APS. ${ }^{35} \mathrm{Na}$ base desses pressupostos, o Ministério da Saúde estabelece incentivos de custeio para a estruturação e implementação de ações de alimentação e nutrição no âmbito da APS pelas secretarias municipais de Saúde e do Distrito Federal, tendo como diretrizes prioritárias a promoção da alimentação adequada e saudável, a Vigilância Alimentar e Nutricional, a prevenção dos agravos relacionados à alimentação e nutrição (sobrepeso e obesidade, desnutrição, anemia por deficiência de ferro, hipovitaminose A, beribéri) e a qualificação da força de trabalho em alimentação e nutrição. 36,37 O Ministério da Saúde também apoia financeiramente a estruturação da Vigilância Alimentar e Nutricional nas unidades básicas de saúde e nos polos do Programa Academia da Saúde, ${ }^{38}$ bem como as ações de prevenção da obesidade infantil em municípios prioritários, no contexto do Programa Saúde na Escola (PSE). ${ }^{39}$ 
Na perspectiva de sistematizar e organizar as ações de alimentação e nutrição e o cuidado nutricional para integrarem o rol de ações desenvolvidas na APS, considera-se que a atuação nesta área deve proceder em dois níveis de intervenção, a gestão das ações e o cuidado nutricional (diagnóstico, promoção da saúde, prevenção de doenças e distúrbios nutricionais, e assistência). Recomenda-se que o cuidado nutricional compreenda tanto abordagens de carácter universal (qualquer fase do curso da vida) como específicas (determinada(s) fase(s) do curso da vida) para os diferentes sujeitos das ações (o indivíduo, a família e a comunidade). ${ }^{35}$ Nesse sentido, a ESF, como espaço privilegiado para o desenvolvimento das ações de alimentação e nutrição e qualificação da atenção à saúde na APS ${ }^{40}$ tem como eixos de assistência nutricional os seguintes: i) promoção de ações integrativas e intersetoriais de educação em saúde e na área de alimentação e nutrição; ii) realização do cuidado nutricional em todas as fases do curso da vida; iii) desenvolvimento de planos terapêuticos para DCNT; iv) intervenção nas deficiências nutricionais; e v) estímulo à produção e ao consumo de alimentos saudáveis. ${ }^{41}$

Apesar da importância reconhecida das ações de nutrição nos serviços de saúde, em geral as mesmas são delegadas a um segundo plano, devido à falta de capacitação dos profissionais responsáveis por seu desenvolvimento. ${ }^{42}$ Segundo relatos profissionais considerando a observação de 20 municípios brasileiros de grande porte, as ações de educação permanente em alimentação e nutrição são escassas como consequência de indisponibilidade de agenda para a realização das capacitações e da falta de profissionais na gestão de tais ações. ${ }^{43}$ Nesse contexto, a inserção do nutricionista na APS, através das equipes dos Núcleos de Apoio à Saúde da Família (NASF), tem por objetivo ampliar a resolubilidade das ações de alimentação e nutrição prestadas pelas equipes da ESF por meio do apoio matricial nas dimensões técnicopedagógicas e clínico-assistenciais. ${ }^{44}$ Permite-se, assim, concretizar o compromisso de integrar a alimentação e nutrição com o setor saúde, em torno da segurança alimentar e nutricional e da garantia do Direito Humano à Alimentação Adequada (DHAA). ${ }^{45}$ Destaca-se, nesse sentido, que o nutricionista é o único profissional com conhecimento específico para realizar diagnóstico nutricional e propor orientações dietéticas necessárias. ${ }^{42}$ Entretanto, sabe-se também que ainda é reduzido o número de nutricionistas que atuam na APS no país, o que pode limitar o desenvolvimento das ações de alimentação e nutrição e criar uma demanda reprimida pelas mesmas, com possíveis consequências relacionadas ao DHAA. ${ }^{46}$

O segundo pilar brasileiro para a Década de Ação em Nutrição inclui a implementação da Estratégia Global para a Alimentação de Lactentes e Crianças de Primeira Infância e do Plano de Ação Global para a Prevenção e Controle de Doenças Não Transmissíveis (2013-2020), definidos pela Organização Mundial da Saúde (OMS). ${ }^{22}$ A Estratégia Global para a Alimentação de Lactentes e Crianças de Primeira Infância tem como intuito definir recomendações relacionadas à duração do aleitamento materno exclusivo e à introdução dos alimentos complementares na idade adequada. ${ }^{47}$ Por sua vez, o Plano de Ação Global para a Prevenção e Controle de Doenças Não Transmissíveis propõe ações de âmbito regional e nacional com o objetivo de reduzir a morbimortalidade, além de minimizar a exposição a fatores de risco e reduzir o ônus socioeconômico dessas doenças. ${ }^{48}$

Nessa perspectiva, no Brasil, a Política Nacional de Alimentação e Nutrição reposicionou a questão alimentar e nutricional na agenda das políticas públicas do setor saúde, enfatizando a importância de práticas alimentares e estilos de vida saudáveis como um componente importante para promoção e proteção da saúde nos primeiros anos de vida. ${ }^{49} \mathrm{Na}$ conjuntura anterior, a Estratégia Amamenta e Alimenta Brasil (EAAB) surgiu como resultado da integração de duas ações importantes do Ministério da Saúde, a Rede Amamenta Brasil e a Estratégia Nacional para a Alimentação Complementar Saudável. ${ }^{50}$ Por sua vez, a Estratégia Intersetorial para Prevenção e Controle da Obesidade direciona esforços para a redução das prevalências de obesidade no país, preocupando-se com a melhoria nos padrões de consumo de alimentos 
e na promoção da atividade física. ${ }^{51}$ Tanto a EAAB ${ }^{52}$ quanto a Estratégia Intersetorial para Prevenção e Controle da Obesidade ${ }^{51}$ apontam o Guia Alimentar para a População Brasileira e o Guia Alimentar para Crianças Menores de Dois Anos como instrumentos técnicos estratégicos de educação alimentar e nutricional da população e como orientadores das ações da saúde para os profissionais e todos os setores envolvidos no sistema alimentar.

Cabe destacar outras ações/estratégias/programas de relevância que formam parte da agenda programática de nutrição na atenção básica à saúde no Brasil. As mesmas incluem a Vigilância Alimentar e Nutricional, que fornece subsídio a diversos sistemas de informação dos quais o Sistema de Vigilância Alimentar e Nutricional é o mais importante; a promoção da alimentação saudável e a prevenção de agravos relacionados à alimentação e nutrição como deficiências nutricionais específicas, com foco na anemia ferropriva e hipovitaminose A, alterações do estado nutricional (desnutrição e excesso de peso) e comorbidades associadas à obesidade. Sendo a intersetorialidade uma das vertentes das ações de alimentação e nutrição, o PBF e o PSE incorporam aspectos importantes relacionados ao cuidado nutricional. ${ }^{53}$ Além disso, outras intervenções estratégicas relacionadas à promoção do crescimento e desenvolvimento da criança também consideram as ações de alimentação e nutrição como componentes essenciais, como o Programa Criança Feliz, ${ }^{54}$ a Estratégia de Atenção Integrada às Doenças Prevalentes na Infância ${ }^{55}$ e a Rede Cegonha. ${ }^{56}$

\section{Proteção social e educação alimentar e nutricional}

A importância da incorporação da agenda de nutrição aos programas de proteção social e de ajuda humanitária, com foco na segurança alimentar e nutricional, é o eixo central do pilar nº 3 dos compromissos brasileiros para a Década de Ação em Nutrição. Estão incluídas nesse pilar políticas de transferência de renda e de doação de alimentos, programas de alimentação escolar e ações de educação alimentar e nutricional. Famílias e pessoas em situação de insegurança alimentar e nutricional, povos e comunidades tradicionais e outros grupos sociais vulneráveis como os beneficiários do PBF conformam os indivíduos prioritários deste compromisso. Para esses grupos, os compromissos do país incluem a oferta de alimentação escolar a 40 milhões de estudantes da rede pública de ensino por ano, sendo 230 mil indígenas e 230 mil quilombolas, a implementação do novo marco legal da Ação de Distribuição de Alimentos, a implementação das recomendações dos guias alimentares para a população brasileira e para crianças menores de dois anos, o subsídio às ações coordenadas e federativas de segurança alimentar e nutricional e de melhorias do estado nutricional das crianças menores de cinco anos e a transferência de renda às famílias em situação de pobreza. ${ }^{22}$

Os Programas de Transferência Condicionada de Renda (PTCR) são considerados uma estratégia de proteção social não contributiva de importância para os ODS, pois buscam erradicar a pobreza extrema e a fome, bem como melhorar a nutrição, a saúde e a educação através das condicionalidades. ${ }^{57}$ Destaca-se, inclusive, sua capacidade de romper a transmissão intergeracional da pobreza ${ }^{57}$ e de integrar políticas setoriais voltadas à população pobre..$^{58}$

Deste modo, os PTCR contribuem com diversos indicadores de capacidades humanas, tais como maior número de crianças e jovens matriculados nas escolas, maior cobertura em medicina preventiva para crianças e melhores indicadores de nutrição infantil. Além disso, tais programas favorecem um maior consumo, pelas famílias beneficiárias, de alimentos e de insumos, como roupas e calçados, sobretudo infantis, promovendo a acumulação de capital humano junto com a redução de carências básicas de bemestar. ${ }^{57}$ Do ponto de vista da nutrição, destaca-se que os PTCR têm efeitos positivos na segurança alimentar 
e nutricional, considerando-se que a transferência possibilita que as famílias comprem maior quantidade de alimentos e privilegiem a qualidade dos mesmos. Além disso, os programas podem influenciar positivamente as visitas preventivas de saúde e a procura de cuidados pré-natais, bem como aumentar a probabilidade de usar saneamento ou fontes de água melhoradas. ${ }^{59}$ Entretanto, os estudos divergem em relação aos impactos na situação vacinal, na ingestão alimentar/diversidade alimentar, no estado de saúde e no crescimento da criança, assim como nos comportamentos do cuidador. Revela-se, nesse sentido, uma lacuna de conhecimento importante relacionada às vias de impacto dos PTCR. ${ }^{59}$

No Brasil, os primeiros PTCR surgiram na década de 90. Em 2003, o PBF foi criado por meio da Medida Provisória n 132, unificando em uma única ação os programas de cunho social propostos anteriormente. 0 PBF propõe contribuir na redução das desigualdades no país e promover a segurança alimentar e nutricional, superando a situação de vulnerabilidade e pobreza, especialmente a extrema. ${ }^{60-62}$

Dentre os programas de transferência monetária existentes no mundo, o PBF é o de maior abrangência. Até meados de agosto de 2014, tinha beneficiado, aproximadamente, 14 milhões de famílias em todos os municípios brasileiros. ${ }^{63} \mathrm{~A}$ atuação do PBF tem como eixos principais: a transferência de renda, que permite aliviar imediatamente a pobreza; as condicionalidades, que reforçam o acesso a direitos básicos na área de educação, saúde e assistência social, e a integração com outras ações e programas complementares que visam ao desenvolvimento das famílias para superação da situação de vulnerabilidade. ${ }^{60}$

A permanência das famílias no Programa está vinculada ao cumprimento das condicionalidades, que envolvem o monitoramento do crescimento e desenvolvimento, o cumprimento do calendário vacinal de crianças menores de sete anos, a realização de consultas de pré-natal nas gestantes, a frequência e assiduidade das crianças nas escolas e a participação nas ações de educação alimentar e nutricional.60,64 As condicionalidades têm por finalidade assegurar às famílias beneficiárias acesso a serviços sociais de qualidade e contribuir na ruptura do ciclo intergeracional de reprodução da pobreza. ${ }^{60}$

O PBF tem proporcionado melhoria nas condições de saúde das crianças beneficiárias. Entre seus impactos destacam-se a redução nas prevalências de insegurança alimentar e nutricional, de baixo-peso ao nascer e de mortalidade relacionada a doenças infecto-contagiosas, desnutrição ou diarreia. Ademais, têm sido postulados efeitos positivos na porcentagem de crianças de até seis meses em amamentação exclusiva, na atualização do calendário vacinal e nas taxas de hospitalização entre menores de cinco anos. ${ }^{65}$ Estudo de âmbito nacional mostrou diminuição dos riscos de desnutrição e de excesso de peso nas crianças menores de cinco anos, por meio do acompanhamento das condicionalidades do Programa. Segundo os resultados, maior tempo de permanência no Programa e a regularidade do acompanhamento em saúde proporcionaram maior probabilidade de evolução nutricional favorável e dentro dos padrões de adequação antropométrica. ${ }^{66}$

Evidências compiladas em revisões sistemáticas sobre o impacto do PBF na segurança alimentar e nutricional e no estado nutricional têm indicado benefícios no acesso aos alimentos em quantidade e variedade, mas sem repercutir necessariamente na qualidade nutricional. Sugere-se que o repasse financeiro suporta, no geral, o acesso a alimentos de baixo valor nutritivo, em detrimento das recomendações de uma alimentação saudável, refletindo no aumento do sobrepeso e da anemia. ${ }^{25,64}$ Este cenário sugere a necessidade de aprimoramento do Programa, ressaltando-se a importância de associar o benefício monetário a ações de educação alimentar e nutricional, uma vez que a transferência isolada de renda não é capaz de resolver o problema da pobreza e da insegurança alimentar e nutricional.25,64,66 Essa perspectiva está implícita no terceiro pilar dos compromissos brasileiros para a Década de Ação em Nutrição, 
ao abordar também a relevância da implementação de ações de informação e educação alimentar e nutricional. ${ }^{22}$

\section{CONSIDERAÇÕES FINAIS}

Além dos determinantes sociais da saúde, as mudanças nutricionais constituem também uma expressão da condição macropolítica, emergindo novos problemas como consequência de uma rotina estressante e intensa, perda de identidade cultural e comprometimento da sustentabilidade ambiental e da biodiversidade. Assim, além dos desafios focados no setor saúde, nos programas de proteção social e nas ações de educação alimentar e nutricional, tratados neste ensaio, são necessárias mudanças estruturais baseadas na redução das iniquidades sociais, como investimentos em educação, reorganização da estrutura fundiária e regulação de mercados internos e externos, com vistas ao interesse de saúde da população e não do mercado. ${ }^{23}$

É importante ressaltar, ainda, que a alimentação adequada e saudável significa também interferir nas repercussões socioambientais do sistema agroalimentar dominante e fortalecer a proposta de soberania alimentar. Não é possível ignorar a ameaça de extinção de 800 tipos de alimentos locais que não são de interesse do setor econômico do agronegócio de exportação, assim como as consequências negativas associadas à alimentação industrializada e ao uso de agrotóxicos, fertilizantes e aditivos sintéticos, drogas veterinárias e produtos provenientes da irradiação. ${ }^{67}$ Adicionalmente, a fragilidade do sistema agroalimentar acentua-se em decorrência da liberalização comercial e da desregulamentação econômica brasileira, que contrasta com o ressurgimento da regulação dos mercados agroalimentares por parte dos Estados nacionais ou blocos regionais como a União Europeia. ${ }^{68}$ Faz-se necessário promover políticas para garantir a disponibilidade e a acessibilidade de alimentos saudáveis, associadas a um ambiente saudável.69,70

\section{CONCLUSÕES}

Apesar dos avanços brasileiros na situação nutricional e a prioridade atual concedida aos problemas nutricionais por meio da Agenda 2030 para o Desenvolvimento Sustentável e da Década de Ação em Nutrição (2016-2025), salienta-se a necessidade de direcionar esforços para grupos em situação de vulnerabilidade que precisam olhar diferenciados, com foco na redução das iniquidades sociais e na promoção do estado nutricional. O período em questão coincide com o enfraquecimento ou desmonte das políticas de proteção social, de saúde e de fortalecimento dos sistemas alimentares sustentáveis e promotores de alimentação saudável, bem como pelo questionamento dos mecanismos de enfrentamento da obesidade, com deterioração das condições de vida e do aumento da pobreza. Nesse sentido, as políticas relacionadas à expansão dos cuidados básicos de saúde e ao aumento do poder aquisitivo/renda familiar nos lares de baixo poder aquisitivo ganham destaque.

A inclusão da nutrição nos sistemas de saúde tem como grande desafio a ampliação do nutricionista na APS, o trabalho interdisciplinar e a capacitação dos profissionais na implantação das ações de alimentação e nutrição; enquanto os programas de proteção social devem promover mudanças estruturais e iniciativas de educação nutricional. Adicionalmente, salienta-se a importância de intervenções como a produção de alimentos saudáveis, a agricultura familiar e o Programa Nacional de Alimentação Escolar, para o alcance das metas da Década de Ação em Nutrição, associadas a mudanças estruturais responsáveis pela má nutrição no país. 


\section{REFERÊNCIAS}

1. World Health Organization. The double burden of malnutrition. Policy brief. Geneva: WHO; 2017.

2. Black RE, Victora CG, Walker SP, Bhutta ZA, Christian $P$, de Onis M, et al. Maternal and child undernutrition and overweight in low-income and middle-income countries. Lancet. 2013; 382(9890): 427-451. http://doi.org/10.1016/S0140-6736(13)60937-X.

3. Cunha AJLA, Leite AJM, Almeida IS. The pediatrician's role in the first Thousand days of the child: the pursuit of healthy nutrition and development. J Pediatr. 2015; 91(Suppl.6): 44-51. http://doi.org/10.1016/j.jped.2015.07.002.

4. Organização Pan-Americana da Saúde. Saúde nas Américas+, Edição de 2017. Resumo do panorama regional e perfil do Brasil. Washington: OPAS; 2017

5. Organização Pan-Americana a Saúde. Desenvolvimento Sustentável e Saúde: tendências dos indicadores e desigualdades no Brasil. Brasília: OPAS; 2014.

6. Programa das Nações Unidas para o Desenvolvimento. Acompanhando a agenda 2030 para o desenvolvimento sustentável: subsídios iniciais do Sistema das Nações Unidas no Brasil sobre a identificação de indicadores nacionais referentes aos objetivos de desenvolvimento sustentável. Brasília: PNUD; 2015.

7. Brasil. Câmara dos Deputados. Conferência das Nações Unidas sobre o Meio Ambiente e Desenvolvimento. Brasília: 1995 [Internet]. [citado em 2019 Mar 20]. Disponível em: http://www.defesacivil.pr.gov.br/arquivos/File/P2R2/agenda21.pdf.

8. Organização das Nações Unidas. Transformando Nosso Mundo: A Agenda 2030 para o Desenvolvimento Sustentável. Brasília: ONU; 2015

9. Brasil. Relatório de Sustentabilidade da. Organização da Conferência das Nações Unidas sobre Desenvolvimento Sustentável. Relatório Rio+20: o Modelo Brasileiro. Brasília: MS; 2012 [Internet]. [citado em 2019 Mar 20]. Disponível em: http://www.rio20.gov.br/documentos/relatorio-rio-20/1.-relatorio-rio-20/at_download/relatorio_rio20.pdf.

10. Organização das Nações Unidas. Relatório sobre os Objetivos de Desenvolvimento do Milénio 2015. New York: ONU; 2015.

11. Brasil. Ministério do Planejamento, Desenvolvimento e Gestão. Relatório Nacional Voluntário sobre os Objetivos do Desenvolvimento Sustentável: Brasil 2017. Brasília: Presidência da República; 2017 [Internet]. [citado em 2019 Mar 20 ]. Disponível em: http://www.secretariadegoverno.gov.br/snas-documentos/relatoriovoluntario_brasil2017port.pdf.

12. Catanante GV, Hirooka LB, Porto HS, Bava MCGGC. Participação social na Atenção Primária à Saúde em direção à Agenda 2030. Ciênc Saúde Coletiva. 2017; 22(12): 3965-974. http://doi.org/10.1590/1413-812320172212.24982017.

13. Bastos ML, Menzies D, Hone T, Dehghani K, Trajman A. The impact of the Brazilian family health strategy on selected primary care sensitive conditions: A systematic review. PLoS One. 2017; 12(8): e0182336. http://doi.org/10.1371/journal.pone.0182336.

14. Brasil. Ministério da Saúde. Gabinete do Ministro. Portaria No 2.436, de 21 de setembro de 2017. Aprova a Política Nacional de Atenção Básica, estabelecendo a revisão de diretrizes para a organização da Atenção Básica, no âmbito do Sistema Único de Saúde (SUS). Diário Oficial da União. 2017 set. 21; Seção 1. p. 68.

15. Ceccon RF, Bueno ALM, Hesler LZ, Kirsten KS, Portes VM, Viecili PRN. Mortalidade infantil e Saúde da Família nas unidades da Federação brasileira, 1998-2008. Cad Saúde Colet. 2014; 22(2): 177-183. http://doi.org/10.1590/1414-462X201400020011.

16. Miranda GMD, Mendes ACG, Silva ALA, Santos Neto PM. A ampliação das equipes de saúde da família e o Programa Mais Médicos nos municípios brasileiros. Trab Educ Saúde. 2017; 15(1): 131-145. http://doi.org/10.1590/1981-7746-sol00051.

17. Gonçalves RF, Sousa IMC, Tanaka OY, Santos CR, Brito-Silva K, Santos LX, et al. Programa Mais Médicos no Nordeste: avaliação das internações por condições sensíveis à Atenção Primária à Saúde. Ciênc Saúde Coletiva. 2016; 21(9): $2815-2824$. http://doi.org/10.1590/1413-81232015219.15392016.

18. Kemper ES, Mendonça AVM, Sousa MF. Programa Mais Médicos: panorama da produção científica. Ciênc Saúde Coletiva. 2016; 21(9): 2785-2796. http://doi.org/10.1590/1413-81232015219.17842016.

19. International Food Policy Research Institute. Global Nutrition Report: From Promise to Impact: Ending Malnutrition by 2030. Washington, DC: IFPRI; 2016.

20. Development Initiatives. Informe de la Nutrición Mundial 2017. "Alimentar los ODS". Bristol, Reino Unido: 2017.

21. World Health Organization. Nutrition. Global targets 2025: Poster [Internet]. [Citado em 2018 Jan 05]. Disponível em: http://www.who.int/nutrition/topics/nutrition_globaltargets2025/en. 
22. Câmara Interministerial de Segurança Alimentar e Nutricional. Compromissos do Brasil para a Década de Ação das Nações Unidas para a nutrição (2016-2025). Brasília: CAISAN; 2015.

23. Souza NP, Lira PIC, Fontbonne A, Pinto FCL, Cesse EAP. A (des)nutrição e o novo padrão epidemiológico em um contexto de desenvolvimento e desigualdades. Ciênc Saúde Coletiva. 2017; 22(7): 2257-2266. http://doi.org/10.1590/141381232017227.03042017

24. Brasil. Ministério do Planejamento, Orçamento e Gestão. Instituto Brasileiro de Geografia e Estatística. Diretoria de pesquisas. Coordenação de trabalhos e rendimentos. Pesquisa Nacional por Amostra de Domicílios: segurança alimentar - PNAD 2013. Rio de Janeiro: IBGE; 2014 [Internet]. [citado em 2019 Mar 20]. Disponível em: http://biblioteca.ibge.gov.br/visualizacao/livros/liv91984.pdf.

25. Cotta RMM, Machado JC. Programa Bolsa Família e segurança alimentar e nutricional no Brasil: revisão crítica da literatura. Rev Panam Salud Publica. 2013; 33(1): 54-60.

26. Bezerra TA, Olinda R, Figueroa Pedraza D. Insegurança alimentar no Brasil segundo diferentes cenários sociodemográficos. Ciênc Saúde Coletiva. 2017; 22(2): 637-651. http://doi.org/10.1590/1413-81232017222.19952015.

27. Brasil. Ministério da Saúde. Pesquisa Nacional de Demografia e Saúde da Criança e da Mulher - PNDS 2006: dimensões do processo reprodutivo e da saúde da criança. Brasília: BVMS; 2009 [Internet]. [citado em 2019 Mar 20]. Disponível em: http://bvsms.saude.gov.br/bvs/publicacoes/pnds_crianca_mulher.pdf.

28. Figueroa Pedraza D, Oliveira MM, Cardoso MVLML, Araujo EMN, Rocha ACD. Índices antropométricos de crianças assistidas em creches e sua relação com fatores socioeconômicos, maternos e infantis. Ciênc Saúde Coletiva. 2016; 21(7): $2219-2232$. http://doi.org/10.1590/1413-81232015217.11712015.

29. Brasil. Ministério da Saúde. Secretaria de Atenção à Saúde. Departamento de Atenção Básica. Política Nacional de Alimentação e Nutrição. 1. ed., 1. reimpr. Brasília: BVMS; 2013 [Internet]. [citado em 2019 Mar 20]. Disponível em: http://bvsms.saude.gov.br/bvs/publicacoes/politica_nacional_alimentacao_nutricao.pdf.

30. Brasil. Ministério do Planejamento, Orçamento e Gestão. Instituto Brasileiro de Geografia e Estatística. Diretoria de Pesquisas. Coordenação de trabalhos e rendimentos. Pesquisa de Orçamentos Familiares: 2008-2009. Antropometria e estado nutricional de crianças, adolescentes e adultos no Brasil. Rio de Janeiro: 2010 [Internet]. [citado em 2019 Mar 20]. Disponível em: http://biblioteca.ibge.gov.br/visualizacao/livros/liv45419.pdf.

31. Meller FO, Araújo CLP, Madruga SW. Fatores associados ao excesso de peso em crianças brasileiras menores de cinco anos. Ciênc Saúde Coletiva. 2014; 19(3): 943-955. http://doi.org/10.1590/1413-81232014193.01552013.

32. Castro IRR. Má nutrição, iniquidade e a garantia do Direito Humano à Alimentação Adequada. Ciênc Saúde Coletiva. 2019; 24(7): 2376-2376. http://dx.doi.org/10.1590/1413-81232018247.15392019.

33. Jaime PC, Delmué DCC, Campello T, Silva DO, Santos LMP. Um olhar sobre a agenda de alimentação e nutrição nos trinta anos do Sistema Único de Saúde. Ciênc Saude Colet. 2018; 23(6): 1829-1836. http://dx.doi.org/10.1590/141381232018236.05392018

34. Monteiro CA, Benicio MH, Konno SC, Silva ACF, Lima AL, Conde WL. Causes for the decline in child under-nutrition in Brazil, 1996-2007. Rev Saude Publica. 2009; 43(1): 35-43. http://dx.doi.org/10.1590/S0034-89102009000100005.

35. Brasil. Ministério da Saúde. Secretaria de Atenção à Saúde. Departamento de Atenção Básica. Matriz de ações de alimentação e nutrição na atenção básica de saúde. Brasília: BVMS; 2009. Série A. Normas e Manuais Técnicos; 78 [Internet]. [citado em 2019 Mar 20]. Disponível em: http://bvsms.saude.gov.br/bvs/publicacoes/matriz_alimentacao_nutricao.pdf.

36. Brasil. Ministério da Saúde. Gabinete do Ministro. Portaria n 1.060, de 24 de maio de 2016. Altera o anexo I e II da Portaria nº 1.738/GM/MS, de 19 de agosto de 2013. Diário Oficial da União. 2016 mai. 24; Seção 1. p. 47.

37. Brasil. Ministério da Saúde. Gabinete do Ministro. Portaria n 55, de 6 de Janeiro de 2017. Estabelece incentivo de custeio para a estruturação e implementação de ações de alimentação e nutrição pelas Secretarias de Saúde dos municípios que possuem população entre 30.000 e 149.999 habitantes (IBGE) e do Distrito Federal, com base na Política Nacional de Alimentação e Nutrição (PNAN). Diário Oficial da União. 2017. jan. 6; Seção 1. p. 25.

38. Brasil. Ministério da Saúde. Gabinete do Ministro. Portaria n².975, de 14 de dezembro de 2011. Apoiar financeiramente a estruturação da Vigilância Alimentar e Nutricional. Diário Oficial da União. 2011 dez. 14; Seção 1. p. 93.

39. Brasil. Ministério da Saúde. Gabinete do Ministro. Portaria n².706, de 18 de outubro de 2017. Lista os Municípios que finalizaram a adesão ao Programa Saúde na Escola para o ciclo 2017/2018 e os habilita ao recebimento do teto de recursos 
financeiros pactuados em Termo de Compromisso e repassa recursos financeiros para Municípios prioritários para ações de prevenção da obesidade infantil com escolares. Diário Oficial da União. 2017 out. 20; Seção 1. p.49.

40. Pimentel VRM, Sousa MF, Hamann EM, Mendonça AVM. Alimentação e nutrição na Estratégia Saúde da Família em cinco municípios brasileiros. Ciênc Saúde Coletiva. 2014; 19(1): 49-58. http://doi.org/10.1590/1413-81232014191.1901.

41. Borelli M, Domene SMA, Mais LA, Pavan J, Taddei JAAC. A inserção do nutricionista na Atenção Básica: uma proposta para o matriciamento da atenção nutricional. Ciênc Saúde Coletiva. 2015; 20(9): 2765-2778. http://doi.org/10.1590/141381232015209.13902014

42. Junqueira TS, Cotta RMM. Matriz de ações de alimentação e nutrição na Atenção Básica de Saúde: referencial para a formação do nutricionista no contexto da educação por competências. Ciênc Saúde Coletiva. 2014; 19(5): 1459-1474. http://doi.org/10.1590/1413-81232014195.11932013.

43. Ricardi LM, Sousa MF. Educação permanente em alimentação e nutrição na Estratégia Saúde da Família: encontros e desencontros em municípios brasileiros de grande porte. Ciênc Saúde Coletiva. 2015; 20(1): $209-218$. http://doi.org/10.1590/1413-81232014201.20812013

44. Fittipaldi ALM, Barros DC, Romano VF. Apoio Matricial nas ações de Alimentação e Nutrição: visão dos profissionais da Estratégia Saúde da Família. Rev Saúde Coletiva. 2017; 27(3): 793-811. http://doi.org/10.1590/s0103-73312017000300021.

45. Alves KPS, Jaime PC. A Política Nacional de Alimentação e Nutrição e seu diálogo com a Política Nacional de Segurança Alimentar e Nutricional. Ciênc Saúde Coletiva. 2014; 19(11): 4331-4340. http://doi.org/10.1590/1413812320141911.08072014.

46. Rigon AS, Schmidt ST, Bógus CA. Desafios da nutrição no Sistema Único de Saúde para construção da interface entre a saúde e a segurança alimentar e nutricional. Cad Saúde Pública. 2016; 32(3): e00164514. http://doi.org/ 10.1590/0102$311 \times 00164514$.

47. World Health Organization; United Nations Children's Fund; International Baby Food Action Network. Estratégia global sobre a alimentação de lactentes e crianças de primeira infância. Revisão de Teresa Setsuko Toma. São Paulo: WHO/UNICEF/IBFAN; 2005.

48. Organização Pan-Americana da Saúde; Organização Mundial da Saúde. Plano de Ação para a Prevenção e Controle de Doenças Não Transmissíveis. Washington: OPAS/OMS, 2013.

49. Recine E, Vasconcellos AB. Políticas nacionais e o campo da Alimentação e Nutrição em Saúde Coletiva: cenário atual. Ciênc Saúde Coletiva. 2011; 16(1): 73-79. http://dx.doi.org/10.1590/S1413-81232011000100011.

50. Einloft ABN, Cotta RMM, Araújo RMA. Promoção da alimentação Saudável na infância: fragilidades no contexto da Atenção Básica. Ciênc Saúde Coletiva. 2018; 23(1): 61-72. http://doi.org/10.1590/1413-81232018231.23522017.

51. Câmara Interministerial de Segurança Alimentar e Nutricional. Estratégia Intersetorial de Prevenção e Controle da Obesidade: recomendações para estados e municípios. Brasília: CAISAN; 2014

52. Brasil. Ministério da Saúde. Gabinete do Ministro. Portaria n 1.920, de 5 de setembro de 2013. Institui a Estratégia Nacional para Promoção do Aleitamento Materno e Alimentação Complementar Saudável no Sistema Único de Saúde (SUS)-Estratégia Amamenta e Alimenta Brasil. Diário Oficial da União. 2013 set. 5; Seção 1. p. 64.

53. Jaime PC, Silva ACF, Lima AMC, Bortolini GA. Ações de alimentação e nutrição na atenção básica: a experiência de organização no Governo Brasileiro. Rev Nutrição. 2011; 24(6): 809-824. http://doi.org/10.1590/S1415-52732011000600002.

54. Brasil. Ministério do Desenvolvimento Social e Agrário. Programa Criança Feliz - A intersetorialidade na visita domiciliar. Brasília: 2017 [Internet]. [citado em $2019 \quad$ Mar 20]. Disponível em: http://www.mds.gov.br/webarquivos/publicacao/crianca_feliz/A_intersetorialidade_na_visita_domiciliar_2.pdf.

55. Brasil. Ministério da Saúde. Organização Pan-Americana da Saúde. Fundo das Nações Unidas para a Infância. Manual de quadros e procedimentos: Aidpi Criança: 2 meses a 5 anos. Brasília: BVMS; 2017 [Internet]. [citado em 2019 Mar 20]. Disponível

em:

http://bvsms.saude.gov.br/bvs/publicacoes/manual_quadros_procedimentos_aidpi_crianca_2meses_5anos.pdf.

56. Brasil. Ministério da Saúde. Secretaria de Atenção à Saúde. Departamento de Atenção Básica. Manual instrutivo das ações de alimentação e nutrição na Rede Cegonha. Brasília: BVMS; 2013 [Internet]. [citado em 2019 Mar 20]. Disponível em: http://bvsms.saude.gov.br/bvs/publicacoes/manual_alimentacao_nutricao_rede_cegonha.pdf. 
57. Organização das Nações Unidas. Comissão Econômica para a América Latina e Caribe. Desenvolvimento sustentável na América Latina e no Caribe: Seguimento da Agenda das Nações Unidas para o Desenvolvimento Pós-2015 e Rio+20. Santiago: ONU; 2013.

58. Neri, M. Uma próxima geração de programas de transferência de renda condicionada. Rev Adm Pública. 2017; 51(2): 168-181. http://dx.doi.org/10.1590/0034-76122017-0041.

59. Groot R, Palermo T, Handa S, Ragno LP, Peterman A. Cash Transfers and Child Nutrition: Pathways and Impacts. Development Policy Review. 2017; 35: 621-643. http://doi.org/10.1111/dpr.12255.

60. Brasil. Ministério do Desenvolvimento Social e Combate à Fome. Bolsa Família: Transferência de renda e apoio à família no acesso à saúde, à educação e à assistência social. Brasília: 2015 [Internet]. [citado em 2019 Mar 20]. Disponível em: http://www.mds.gov.br/webarquivos/publicacao/bolsa_familia/Cartilhas/Cartilha_PBF_2015.pdf.

61. Monteiro F, Schmidt ST. O Programa Bolsa Família no contexto da segurança alimentar e nutricional no Brasil. Sau. \& Transf. Soc. 2014; 5(3); 91-97.

62. Martins APB, Canella DS, Baraldi LG, Monteiro CA. Transferência de renda no Brasil e desfechos nutricionais: revisão sistemática. Rev Saúde Pública. 2013; 47(6): 1159-1171. http://doi.org/10.1590/S0034-8910.2013047004557.

63. Silva ESA, Paes NA. Programa Bolsa Família e Mortalidade Infantil no Brasil: Revisão Integrativa. Holos. 2018; 1: $201-211$. http://doi.org/10.15628/holos.2018.4836.

64. Wolf MR, Filho AA. Estado nutricional dos beneficiários do Programa Bolsa Família no Brasil - uma revisão sistemática. Ciênc Saúde Coletiva. 2014; 19(5): 1331-1338. http://doi.org/10.1590/1413-81232014195.05052013.

65. Campello T, Neri MC. Uma década derrubando mitos e superando expectativas. In: Instituto de Pesquisa Econômica Aplicada. Programa Bolsa Família: uma década de inclusão e cidadania. Brasília: IPEA; 2014 [Internet]. [citado em 2019 Mar 20 ]. Disponível em: http://www.ipea.gov.br/portal/images/stories/PDFs/livros/livros/livro_bolsafamilia_10anos.pdf.

66. Brasil. Ministério da Saúde. Ministério do Desenvolvimento Social e Combate à Fome. Avaliação da evolução temporal do estado nutricional das crianças do Programa Bolsa Família (PBF), acompanhadas nas condicionalidades de saúde. Brasília: $2014 \quad$ [Internet]. [citado em $2019 \quad$ Mar 20]. Disponível em: http://aplicacoes.mds.gov.br/sagirmps/simulacao/sum_executivo/pdf/sumario_138.pdf.

67. Azevedo E, Ribas MTGO. Estamos seguros? Reflexões sobre indicadores de avaliação da segurança alimentar e nutricional. Rev Nutr. 2016; 29(2): 241-251. http://dx.doi.org/10.1590/1678-98652016000200008.

68. Ribeiro CSG, Pilla MCBA. Segurança alimentar e nutricional: interfaces e diminuição de desigualdades sociais. Demetra. 2014; 9(1): 41-52. http://dx.doi.org/10.12957/demetra.2014.6642.

69. Haire-Joshu D, Tabak R. Preventing Obesity Across Generations: Evidence for Early Life Intervention. Annu Rev Public Health. 2017; 37: 253-271. doi: 10.1146/annurev-publhealth-032315-021859.

70. Jaacks LM, Kavle J, Perry A, Nyaku A. Programming maternal and child overweight and obesity in the context of undernutrition: current evidence and key considerations for low- and middle-income countries. Public Health Nutr. 2017; 20(7): 1286-1296. doi: $10.1017 / S 1368980016003323$

\section{Colaboradores}

Figueroa Pedraza D participou da concepção do artigo, revisão bibliográfica, análise e interpretação dos dados, redação e revisão final do artigo; Lins ACL, Santos EES e Oliveira MM participaram da revisão bibliográfica, análise e interpretação dos dados, redação e revisão final do artigo.

Conflito de Interesses: Os autores declaram não haver conflito de interesses.

Recebido: 05 de junho de 2019

Aceito: 27 de fevereiro de 2020 